\section{Muscular activation during reverse and non-reverse chewing cycles in unilateral posterior crossbite}

\author{
Piancino MG, Farina D, Talpone F, Merlo A, Bracco P. Muscular activation during \\ reverse and non-reverse chewing cycles in unilateral posterior crossbite. Eur J Oral Sci \\ 2009; 117: 122-128. (c) 2009 The Authors. Journal compilation (c) 2009 Eur J Oral Sci
}

The aim of this study was to characterize the kinematics and masseter muscle activation in unilateral posterior crossbite. Eighty-two children $(8.6 \pm 1.3 \mathrm{yr}$ of age) with unilateral posterior crossbite and 12 children $(8.9 \pm 0.6 \mathrm{yr}$ of age) with normal occlusion were selected for the study. Electromyography (EMG) and kinematics were concurrently recorded during mastication of a soft bolus and a hard bolus. The percentage of reverse cycles in the group of patients was $59.0 \pm 33.1 \%$ (soft bolus) and $69.7 \pm 29.7 \%$ (hard bolus) when chewing on the crossbite side. When chewing on the non-affected side, the number of reverse cycles was $16.7 \pm 24.5 \%$ (soft bolus) and $16.7 \pm 22.3 \%$ (hard bolus). The reverse cycles on the crossbite side were narrower with respect to the cycles on the non-affected side. Although both types of cycles in patients resulted in lower EMG activity of the masseter of the crossbite side than of the contralateral masseter, the activity of the non-affected side was larger for reverse than for non-reverse cycles. It was concluded that when chewing on the crossbite side, the masseter activity is reduced on the mastication side (crossbite) and is unaltered (nonreverse cycles) or increased (reverse) on the non-affected side.
Maria Grazia Piancino ${ }^{1}$, Dario Farina $^{2}$, Francesca Talpone ${ }^{1}$, Andrea Merlo', Pietro Bracco ${ }^{1}$

${ }^{1}$ Department of Orthodontics and Gnathology, Dental School, Turin University, Turin, Italy; ${ }^{2}$ Center for Sensory-Motor Interaction (SMI), Department of Health Science and Technology, Aalborg University, Aalborg, Denmark

Maria Grazia Piancino, PhD, Department of Orthodontics, Turin University, V. Nizza 230, I-10126 Torino, Italy

Telefax: +39-011-885414

E-mail: mpianci@tin.it

Key words: chewing pattern; crossbite; jaw muscles; reverse chewing cycle; surface EMG

Accepted for publication November 2008
Mastication is one of the most complex and co-ordinated functional movements. The masticatory system is capable of exerting high forces through the execution of very precise movements. The accuracy of the movement is important for avoiding damage to the stomathognatic system and for maximizing efficiency. The pattern of mandibular movement during chewing is influenced by factors such as the bolus type and the type of occlusion (1-4). The relative position of the upper and lower teeth determines occlusal stability, which is related to muscular performance.

Crossbite is a widespread malocclusion, prevalent in the range of $8-22 \%$ (5). Unilateral posterior crossbite, which represents half of all crossbites, is an asymmetric malocclusion characterized by an inverse relationship of the upper and lower buccal dental cusps, in the molar and premolar regions, on one side only of the dental arch. It may originate from a skeletal relationship or a dental relationship, or both, and may lead to mandibular displacement accompanied by lower midline deviation (6). It develops in children between 2 and $5 \mathrm{yr}$ of age, during eruption of the primary dentition, and can involve the permanent dentition at a later stage of development $(7,8)$.

Compared with control groups, children with unilateral posterior crossbite exhibit different kinematics of the mandible during mastication when chewing on the affected side. During normal chewing, the mandible deviates laterally towards the bolus side and then medially during closure (9). The frequency of reverse chewing cycles is substantially increased in children with unilateral posterior crossbite $(1,10-14)$. In reverse cycles the mandible first deviates medially and then laterally, thus ensuring overlap of opposing dental occlusal surfaces. Reverse chewing cycles show an abnormal, narrow pattern characterized by smaller lateral displacement and slower velocity of the mandible in comparison with normal chewing. In a patient with unilateral crossbite, reverse cycles occur mainly on the crossbite side, although not all cycles are reverse when chewing from the crossbite side (11-16).

Mandibular movements during chewing depend on the interplay of the masticatory muscles; thus, abnormal chewing kinematics is associated with altered muscle activation. Abnormal chewing in children has an impact on the growing structures and may lead to an irreversible asymmetry of the anatomical structures (bones, temporomandibular joint, muscles, teeth) (17-21).

In patients with unilateral posterior crossbite, the masseter of the crossbite side is less active $(22,23)$, and the co-ordination of the masticatory muscles on the two sides is altered (24), with respect to controls. However, no studies have investigated concurrently the kinematics of the chewing cycle and the electromyography (EMG) activity of masticatory muscles in patients with unilateral crossbite. Moreover, it is not known if reverse and 
non-reverse cycles, when chewing from the crossbite side, result in different coordination among muscles of the two sides. This knowledge is important for understanding the muscular load in patients with unilateral crossbite because abnormal loads may result in long-term changes in the bite force (25), muscle cross-section (26), and in other structures. Moreover, the dental therapy aimed at correcting the malocclusion should aim to achieve a functional improvement, for which it is necessary to understand in greater detail the neuromuscular adaptations associated with the malocclusion.

In this study, it was hypothesized that the kinematics and muscular activity associated with both the reverse and non-reverse chewing cycles on the crossbite side are different from the cycles on the non-affected side. Therefore, the aim of this study was to characterize the reverse and non-reverse chewing cycles in both the affected and non-affected sides of patients with unilateral posterior crossbite.

\section{Material and methods}

Eighty-two children, $8.6 \pm 1.3$ yr of age [mean \pm standard deviation (SD)], with unilateral posterior crossbite (50 on the right side, 32 on the left side) and 12 children $8.9 \pm 0.6 \mathrm{yr}$ of age (mean $\pm \mathrm{SD}$ ), with normal occlusion, were selected for the study out of 1,200 patients referred to the Orthodontic Department of the University of Turin in the period December 2005 to December 2006. Before participating in the study, informed consent was obtained from the parents.

The inclusion criteria for the patient group were: (i) unilateral posterior crossbite of two or more posterior teeth, (ii) mixed dentition, (iii) no erupting teeth, (iv) no teeth to be substituted, (v) eight permanent incisors, four deciduous canines, eight deciduous molars, four permanent molars, (vi) no caries, and (vii) no pain. The exclusion criteria were the presence of (i) any previous orthodontic therapy, (ii) any signs or symptoms of dental or myofacial pain, (iii) any signs of cranio-mandibolar disorders, or (iv) any prosthesis. The control group was strictly selected for normal occlusion and mixed dentition, and was matched with the patient group for age and gender.

\section{Procedures}

The children were comfortably seated on a chair. They were asked to fix their eyes on a target (a red beak of a Donald Duck drawing) on the wall, $90 \mathrm{~cm}$ directly in front of their seating position, and to avoid movements of the head. The measures were performed in a silent and comfortable environment. Each recording began with the largest number of teeth in contact. The children were asked to find this starting position by lightly tapping their opposing teeth together and clenching. They were asked to hold this position with the test bolus on the tongue, prior to starting the recording. Each recording consisted of chewing for a time-period of $10 \mathrm{~s}$ and was repeated, for each experimental session, three times for mastication on the right side and three times for mastication on the left side, using a soft bolus and a hard bolus. An operator controlled (visual inspection) the side of mastication. The soft bolus was a piece of chewing gum and the hard bolus was a wine gum, both of which were the same size $(20 \mathrm{~mm}$ in length, $1.2 \mathrm{~mm}$ in height, and $0.5 \mathrm{~mm}$ in width) but of different weights ( $2 \mathrm{~g}$ for the soft bolus and $3 \mathrm{~g}$ for the hard bolus). The wine gum was chosen to provide a rubber-like resistance without sticking to the teeth.

\section{Kinematic analysis}

The mandibular motion was tracked using a kinesiograph (K6-I; Myotronics, Tukwila, WA, USA) that measures jaw movements with an accuracy of $0.1 \mathrm{~mm}$. Multiple sensors (Hall effect) in a light-weight (113 g) array tracked the motion of a tiny magnet attached at the lower interincisor point. The kinesiograph was interfaced with a computer for data storage and subsequent analysis.

\section{EMG recordings}

Surface EMG signals were recorded from the masseter muscles of both sides using a multichannel electromyograph (bandwidth 45-430 Hz per channel; Myotronics). This EMG amplifier is part of the K6-I WIN Diagnostic System (27). The relatively large high-pass frequency in EMG recordings was selected to reduce low-frequency movement artifacts during chewing. Two electrodes (Duotrode silver/ silver chloride EMG electrodes; Myotronics) were located on the masseter and temporalis anterior muscles of both sides with an interelectrode distance of $20 \mathrm{~mm}$. Before electrode placement, the skin was lightly abraded with abrasive paste and cleaned with ethanol. The location of the electrodes was based on anatomical landmarks (28). Kinetic and EMG data were recorded concurrently.

\section{Signal analysis}

The kinematic signals were analyzed using custom-made software (Department of Orthodontics and Gnathology, Dental School, Turin University, Turin, Italy). The first cycle, during which the bolus was transferred from the tongue to the dental arches, was excluded from the analysis. Other cycles were excluded if they presented at least one of the following characteristics: (i) minimum opening smaller than $4 \mathrm{~mm}$; (ii) duration shorter than $300 \mathrm{~ms}$; or (iii) vertical opening smaller than $3 \mathrm{~mm}$.

From each cycle, the following variables were extracted (9): (i) opening amplitude in the frontal plane; (ii) closure angle; (iii) cycle duration; (iv) maximum lateral excursion; and (v) maximum velocity (in the three-dimensional space). The variable values computed for each included cycle were averaged for cycles recorded for the same side of mastication.

The chewing cycles were divided into non-reverse and reverse, based on the vectorial direction of closure. The closure angle was measured from the horizontal line of the side of mastication. Cycles with a closure angle larger than $90^{\circ}$ were grouped in the reverse set. The surface EMG was rectified and low-pass filtered with a $10 \mathrm{~Hz}$ cut-off frequency (signal envelope). The maximum value of the EMG envelope was extracted to quantify the peak in muscle activity during the chewing cycle.

\section{Statistical analysis}

Kinematic and EMG data from the control group were analyzed using repeated-measures analysis of variance (ANOVA) to assess a potential influence of the side as the 
main factor. The kinematic and EMG variables of the nonreverse cycles of the control group were compared with the same variables of the non-reverse cycles of the non-affected side of the patient group using a mixed-model ANOVA with the subject group (patients or controls) as the betweengroup factor and with the bolus hardness and (for the surface EMG only) the muscle side as within-group factors. The non-reverse cycles of the non-affected and crossbite side of the patient group, and the reverse and non-reverse cycles of the patient group, were compared using ANOVA. When ANOVA was significant, pair-wise comparisons were tested with the post-hoc Student-Newman-Keuls (SNK) test. The significance level was $P<0.05$.

\section{Results}

In the group of patients, the percentage of reverse cycles when chewing on the crossbite side was $59.0 \pm 33.1 \%$ (soft bolus) and $69.7 \pm 29.7 \%$ (hard bolus). When chewing on the non-affected side, the number of reverse cycles was $16.7 \pm 24.5 \%$ (soft bolus) and $16.7 \pm 22.3 \%$ (hard bolus). The control subjects yielded $4.7 \pm 5.9 \%$ (soft bolus) and $2.1 \pm 3.3 \%$ (hard bolus) reverse cycles (both sides pooled together), which were excluded from the analysis.

\section{Kinematics}

Figure 1 shows an example of reverse and non-reverse cycles in a patient compared with a control subject. In the control group, a two-way ANOVA, with factors the side of mastication and the bolus hardness, was not significant for the side of mastication for all kinematic variables. The closure angle $(F=4.7, P<0.05)$, opening amplitude $(F=19.4, \quad P<0.01)$, maximum lateral excursion $(F=9.3, P<0.05)$, and maximum velocity $(F=27.6$, $P<0.001)$ were dependent on the bolus hardness (Table 1), as shown previously (9). Because the side of mastication did not affect the results in the control group, in this group the kinematic variables were averaged over the two sides for subsequent analysis.

The non-reverse chewing cycles of the non-affected side of the patients were compared with the chewing cycles of the control group using a two-way ANOVA with factors the group and the bolus hardness. The dependency on bolus hardness for the patient group was the same as for the control group. Only the opening amplitude was different among the two groups $(F=5.6$, $P<0.05)$, with larger values observed for the controls $(P<0.05$; Table 1$)$.

The non-reverse cycles of the patients were compared among the two sides using a two-way ANOVA with factors the side (crossbite side and non-affected side) and the bolus hardness. The closure angle, maximum lateral excursion, and duration were dependent on the side $(F=23.3, P<0.0001 ; F=16.0, P<0.001 ; F=7.9$, $P<0.01$; Table 1). Thus, the cycles classified as non-reverse according to the closure characteristics had different kinematics in the two sides.

Finally, the reverse and non-reverse cycles when chewing on the crossbite side were compared using a two-way ANOVA with factors the type of cycle and the bolus hardness. The closure angle was different in the two types of cycles as the reverse cycles were defined on the basis of the closure angle. In addition, the maximum lateral excursion was larger for the non-reverse cycle compared with the reverse cycle (ANOVA: $F=28.5$, $P<0.00001$; SNK: $P<0.001$; Table 1).

In summary, the kinematic data indicated that the non-reverse cycles on the non-affected side differed from the cycles of the control group only for the opening amplitude. The non-reverse cycles in the patients were different among sides. The reverse and non-reverse cycles in the patients (crossbite side) differed by closure angle (inversion in closure) and by maximum lateral excursion, indicating that the reverse cycles were narrower than the non-reverse cycles.

\section{Muscle activity}

The chewing cycles of the control subjects were analyzed for excluding a dependency on the side of mastication using a three-way ANOVA with factors the side of mastication, the muscle side, and the bolus hardness. None of the EMG variables were dependent on the side of mastication as the main factor, and thus the results from the two sides were averaged for the control group.

The non-reverse cycles of the non-affected side in the patient group were compared with the chewing cycles of the control group using a three-way ANOVA with factors the group, the muscle side, and the bolus hardness. The peak EMG did not depend on the group of subjects (patients/controls), indicating that the non-reverse cycles of the non-affected side of patients had muscular activity similar to that of controls. The dependency on the bolus hardness and the muscle side confirmed previous work (9): the peak EMG of the side of mastication was larger than in the contralateral side $(P<0.01)$ and was larger for the hard bolus than for the soft bolus $(P<0.001)$.

The non-reverse cycles were compared between the two sides of the patients using a three-way ANOvA with factors the side of mastication, the muscle side, and the bolus hardness. The peak EMG amplitude was dependent on the side of mastication $(F=9.1, P<0.01)$, on the bolus $(F=78.4, P<0.0001)$, and on the interaction between the side of mastication and the muscle side $(F=170.3, P<0.0001)$. The peak EMG was larger when the mastication was on the non-affected side ( $P<0.001$ for both muscles) but the difference was only seen for the muscle on the side of mastication [i.e. the masseter of the crossbite side when chewing on the crossbite side was less active than the masseter of the non-affected side when chewing on the non-affected side $(P<0.01$ for both muscles)]. This indicated a reduced activity of the masseter when chewing on the crossbite side, even for non-reverse cycles.

The reverse and non-reverse cycles for the affected side were compared in the patient group using a three-way ANOVA with factors the type of cycle, the muscle side, and the bolus hardness. The peak EMG activity was dependent on the type of cycle $(F=6.2, P<0.05)$, the muscle side $(F=27.6, P<0.0001)$, the bolus hardness 


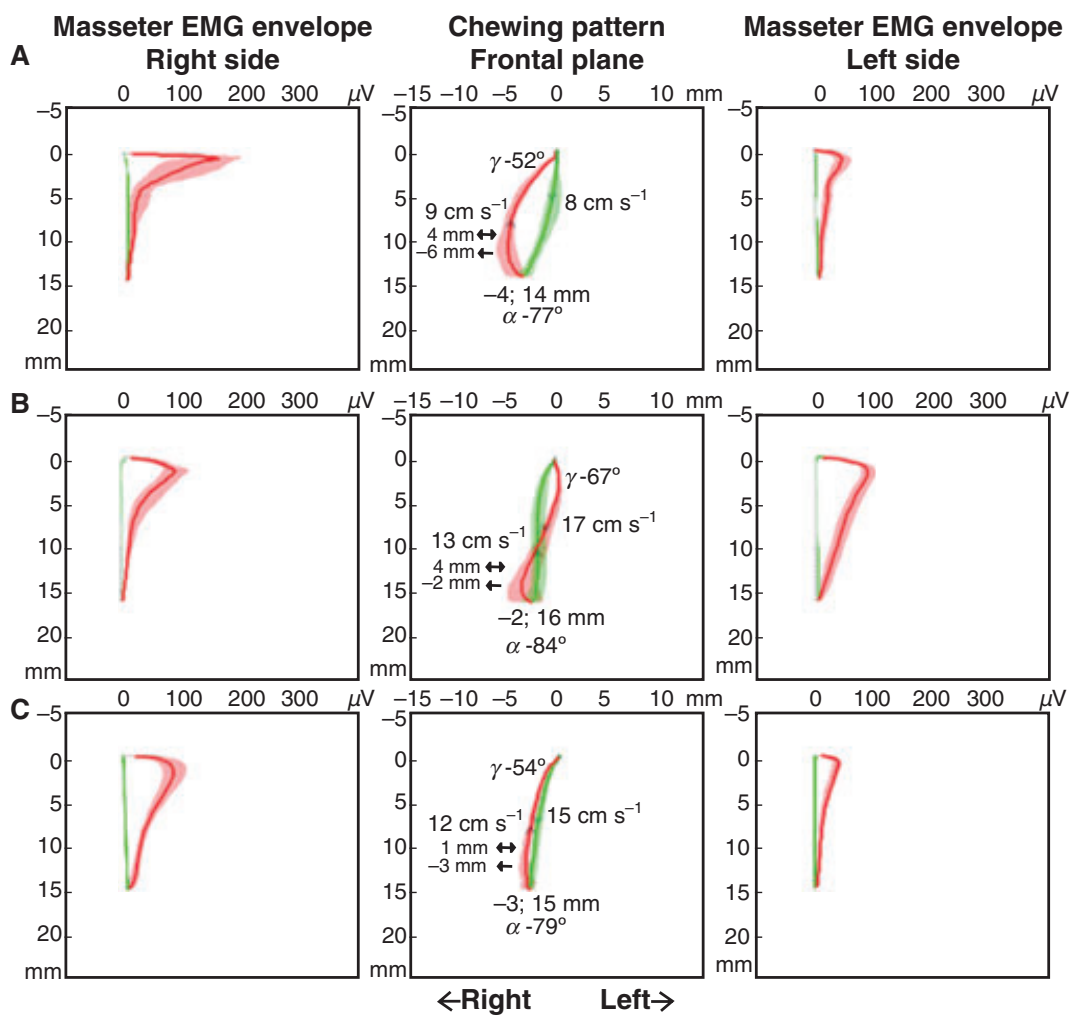

Fig. 1. (A) Average chewing cycle and muscular activity, during chewing, on the right side of a control subject. The horizontal lines represent the standard deviation over the averaged cycles. The chewing pattern is right oriented, the closure direction is on the right, and the masseter of the right side (the side of the bolus) is more active than the masseter of the contralateral side. (B) Non-reverse chewing cycle in a patient when chewing on the crossbite side (left). The direction of closure is correct (non-reverse) but the pattern is narrower with respect to the control. The activity of the right masseter is lower in comparison with normal mastication. (C) Reverse chewing cycle in the same patient shown in (B). The direction of closure is reverse, the pattern is highly anomalous, and the right masseter is less active than in the control, whereas the left masseter is more active than in the control.

Table 1

Kinematic variables (mean \pm standard deviation) for the control group and the patient group (non-affected side)

\begin{tabular}{lccccc}
\hline & \multicolumn{2}{c}{ Control } & & \multicolumn{2}{c}{$\begin{array}{c}\text { Non-reverse, mastication on } \\
\text { non-affected side }\end{array}$} \\
\cline { 2 - 3 } & Soft bolus & Hard bolus & & Soft bolus & Hard bolus \\
\hline Closure angle (degrees) & $60.2 \pm 8.4$ & $56.4 \pm 9.4$ & & $64.2 \pm 12.3$ & $61.8 \pm 11.4$ \\
Opening amplitude (mm) & $14.7 \pm 3.6$ & $18.4 \pm 5.1$ & & $12.5 \pm 3.7$ & $15.0 \pm 4.1$ \\
Maximum lateral excursion (mm) & $3.9 \pm 0.9$ & $4.3 \pm 0.9$ & $4.0 \pm 2.2$ & $4.0 \pm 2.0$ \\
Duration (ms) & $602.5 \pm 102.9$ & $582.6 \pm 81.2$ & $546.8 \pm 105.3$ & $544.4 \pm 95.2$ \\
Maximum velocity (mm s${ }^{-1}$ ) & $120.3 \pm 4.5$ & $150.4 \pm 4.2$ & & $100.8 \pm 3.3$ & $130.2 \pm 4.3$ \\
\hline
\end{tabular}

$(F=102.0, \quad P<0.0001)$, and on the interaction between the type of cycle and the muscle side $(F=11.4$, $P<0.01$ ). The non-affected side (contralateral to the mastication) was less active than the crossbite side $(P<0.001$; Fig. 2). Moreover, the activity of the nonaffected side was higher in the reverse cycles than in the non-reverse cycles $(P<0.001$; Fig. 2$)$, whereas there was no difference in activity of the crossbite side between the two types of cycles.

In summary, the EMG data indicated that in the patients and controls, similar muscular activation occurred in the non-reverse cycles of the non-affected side. However, the non-reverse cycles were different between the two sides in the patients, with lower EMG amplitude observed for the muscle on the side of mastication when chewing on the crossbite side. The reverse and non-reverse cycles of the patient group (crossbite side) differed in activity of the masseter of the side contralateral to the mastication (non-affected side), which was more active in reverse cycles, but not in activity of the masseter of the crossbite side. Thus, mastication on the crossbite side was characterized by a decreased activity of the masseter on the side of mastication for both reverse and non-reverse cycles and by an increased 

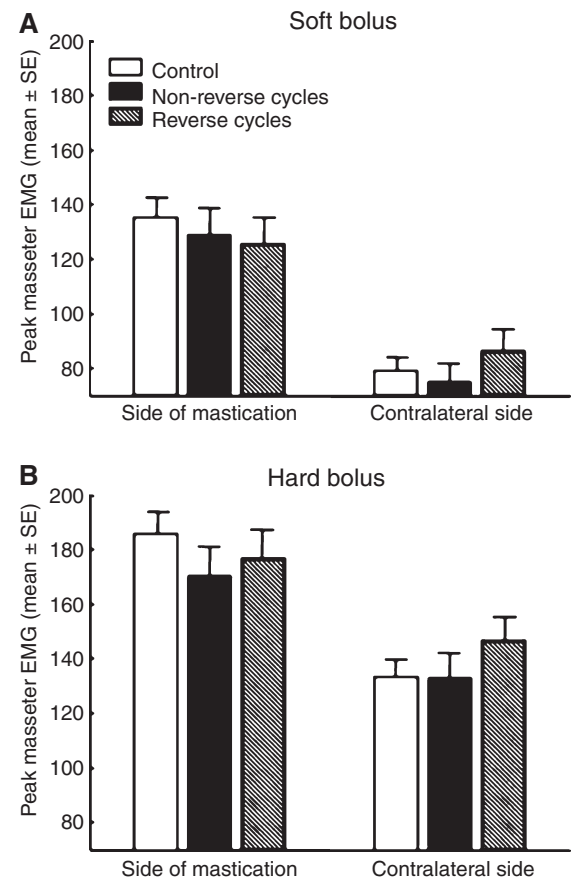

Fig. 2. (A) Electromyography (EMG) peak envelope [mean \pm standard error (SE)] for the control group (two sides averaged) and the patient group during mastication (of the soft bolus) on the crossbite side for reverse and non-reverse cycles. (B) Same data as in (A) but during mastication of the hard bolus.

activity of the masseter on the contralateral side only when the cycle was reverse.

\section{Discussion}

The kinematic and EMG characteristics of the masseters of chewing cycles from the non-affected side of patients with unilateral posterior crossbite were similar to those of controls. The chewing cycles, both reverse and nonreverse, on the crossbite side for the patient group, showed different characteristics with respect to controls, and were all characterized by a decreased activity of the masseter of the crossbite side. Reverse cycles were additionally characterized by increased contralateral activity with respect to non-reverse cycles.

The generation of the chewing pattern is based on inputs from the peripheral receptors that are directly connected to the mesencephalic trigeminal nucleus (2933 ) and to the trigeminal motor neurons and interneurons (34). The crossbite of the primary dentition has been defined as 'positional or functional crossbite' $(7,35)$; at this stage the mandible can still close in a centric position but the edge-to-edge dental contacts shift the mandible on one side to reach a stable occlusal position. The edgeto-edge contacts send inputs to the central nervous system, which adapts the chewing motor scheme while maintaining the efficiency of mastication $(31,36)$.

Only a few studies have investigated the EMG activity of the masseter muscle in patients with unilateral crossbite during chewing on the non-affected side (24). The results of the present study are in accordance with previous results and indicate that the masseters of the two sides, when chewing on the non-affected side, have activity similar to that of the control group. This reflects a normal kinematics of the chewing cycle in the nonaffected side of patients (14).

When chewing on the crossbite side, the reverse chewing cycles are prevalent, but some non-reverse chewing cycles are still present $(14,15)$. In this study the chewing cycles of the crossbite side were divided into reverse and non-reverse because the percentage of non-reverse cycles was sufficient for separate statistical analysis. The examination of both types of cycles on the crossbite side is relevant for understanding the muscular activation during daily chewing in relation to the percentage of the two types of cycles. It is known that reverse chewing cycles are substantially different from normal cycles; reverse cycles are narrow, the closure trajectory is near the vertical line or it may be displaced on the opposite side of the bolus, and the opening and closing trajectories may cross each other (14). There are no previous data on the kinematics and muscular activation during both reverse and non-reverse cycles on the crossbite side.

The non-reverse cycles on the crossbite side were characterized by a smaller lateral displacement, reduced opening, and larger closure angle in comparison to controls. These cycles were thus less efficient, according to the efficiency criteria (height, width, angle of closure) described by WILDING \& LEWIN (2), even if the direction of closure was normal. Despite the different kinematics, the masseter muscles of the two sides maintained an asymmetric activation depending on the side of mastication, which is likely to be a basic feature of masticatory muscle activity. Moreover, both sides increased their activity when the bolus hardness was increased, as in control subjects $(9,37)$. The co-ordinated activity of both sides, with the side of the bolus more active than the other, is a characteristic of mastication that is maintained under various conditions. For example, edentulous patients with new complete dentures maintain this co-ordination, despite a large decrease in muscle activation (38). Thus, although anatomical alterations are present, the central nervous system maintains this co-ordinated motor scheme, which is one of the oldest phylogenetic patterns.

Although the masseter of the mastication side was more active than the masseter of the contralateral side during all cycles, the masseter of the crossbite side was less active than in controls for both reverse and nonreverse cycles. The reverse cycles also presented increased activity of the masseter of the non-affected side. Thus, while mastication from the non-affected side was normal, mastication from the crossbite side was associated with a reduced activity of the masseter of the crossbite side and unaltered (non-reverse) or increased (reverse) activation of the masseter of the non-affected side. As a consequence, in the long term the masseter muscle of the nonaffected side will be substantially more loaded than the masseter muscle of the crossbite side and the overload will be dependent on the percentage of reverse cycles. 
This activation asymmetry is harmful for growing structures, such as muscles, bones, and joints $(17,18,21)$. In accordance with our results, it has been recently shown that the masseter of the crossbite side in children with unilateral crossbite has a lower bite force (25), and a smaller cross-sectional area (26), than the masseter of the non-affected side. The abnormal muscle activation observed in this study may explain these functional and anatomical asymmetries. These results indicate that the altered muscle activation should be corrected to avoid potentially permanent changes of the muscles and bones (7, 17, 39).

The reduction in percentage of reverse cycles decreases the alteration of muscle activation, with respect to normal mastication, by reducing the overload on the nonaffected side. This should thus be a primary aim of therapeutic interventions. A functional correction is, however, not always achieved using current orthodontic therapies, even when the anatomical alteration (malocclusion) is corrected $(5,39,40)$. Indeed, it has been reported in many studies that the percentage of reverse chewing cycles does not decrease substantially after dental correction (11-14). The difficulty in correcting the chewing cycles might be related to the fact that this malocclusion develops during the eruption of the primary dentition when the chewing pattern is still not developed (14). Recently, it has been shown that a functional appliance corrects the dental malocclusion and substantially decreases the number of reverse chewing cycles after therapy. The kinematic/EMG analysis after correction of the malocclusion should thus be the basis for correct interventions (15).

As a limitation of the study, surface EMG measures are affected by factors other than the intensity of muscle activity (e.g. the thickness of the subcutaneous tissue layer and cross-talk). These factors may have increased the variability of the data across subjects. Moreover, the repeatability of the data may have been poorer for the patient group with respect to the control group.

In conclusion, the results show that muscle activity during mastication on the non-affected side is normal, whereas on the crossbite side muscle activity during mastication is altered both in reverse and non-reverse cycles. The masseter of the non-affected side is, in general, more loaded than in healthy controls, whereas the masseter of the crossbite side is less active. The amount of overload of the non-affected side depends on the percentage of reverse cycles. The altered muscular activation corresponds to altered kinematics of the reverse chewing cycles. The reduction in the percentage of reverse cycles is important for decreasing the altered muscular activity because it reduces the overload of the non-affected side. A functional correction of the chewing cycles should be an important aim of the orthodontic therapy.

\section{References}

1. LEWIN A. Electrognathographics: atlas of diagnostic procedures and interpretation. Berlin: Quintessence, 1985; 82-85.
2. Wilding RJ, Lewin A. The determination of optimal human jaw movements based on their association with chewing performance. Arch Oral Biol 1994; 39: 333-343.

3. Woda A, Foster K, Mishellany A, Peyron MA. Adaptation of healthy mastication to factors pertaining to individual or to the food. Physiol Behav 2006; 89: 28-35.

4. Ferrario VF, Piancino MG, Dellavia C, Castroflorio T, SFOrZa C, Bracco P. Quantitative analysis of the variability of unilateral chewing movements in young adults. Cranio 2006; 24: 274-282.

5. Harrison JE, Ashby D. Orthodontic treatment for posterior crossbites. Cochrane Database Syst Rev 2000; 1: CD000979.

6. Daskalogiannakis J. Glossary of orthodontic terms. Berlin: Quintessence Publishing Group, 2002.

7. Nerder PH, Bakke M, Solow B. The functional shift of the mandible in unilateral posterior crossbite and the adaptation of the temporomandibular joints: a pilot study. Eur J Orthod 1999 21: $155-166$.

8. Thilander B, Lennartsson B. A study of children with unilateral posterior crossbite, treated and untreated, in the deciduous dentition - occlusal and skeletal characteristics of significance in predicting long term outcome. J Orofac Orthop 2002; 63: 371-383.

9. Piancino MG, Bracco P, Vallelonga T, Merlo A, Farina D. Effect of bolus hardness on the chewing pattern and activation of masticatory muscles in subjects with normal dental occlusion. J Electromyogr Kinesiol 2008; 18: 931-937.

10. Ahlgren J. Pattern of chewing and malocclusion of teeth. A clinical study. Acta Odontol Scand 1967; 25: 3-13.

11. Ben-Bassat Y, Yaffe A, Brin I, Freeman J, Ehrlich Y. Functional and morphological occlusal aspects in children treated for unilateral posterior cross-bite. Eur J Orthod 1993; 15: $57-63$

12. Brin I, Ben Bassat Y, Blustein Y, Ehrlich Y, Hochman N, MARMARY Y, YAFFE A. Skeletal and functional effects of treatment for unilateral posterior crossbite. Am J Orthod Dentofacial Orthop 1996; 109: 173-179.

13. Saitoh I, Hayasaki H, Iwase Y, Nakata M. Improvement of jaw motion following treatment of unilateral crossbite in a child with primary dentition: a case report. Cranio 2002; 20: 129-134.

14. Throckmorton GS, Buschang PH, Hayasaki H, Pinto AS Changes in the masticatory cycle following treatment of posterior unilateral crossbite in children. Am J Orthod Dentofacial Orthop 2001; 120: 521-529.

15. Piancino MG, Talpone F, Dalmasso P, Debernardi C, LEWIN A, BRACCO P. Reverse-sequencing chewing patterns before and after treatment of children with unilateral posterior crossbite. Eur J Orthod 2006; 28: 480-484.

16. Martin C, Alarcon JA, Palma JC. Kinesiographic study of the mandible in young patients with unilateral posterior crossbite. Am J Orthod Dentofacial Orthop 2000; 118: 541-548.

17. Moller E, Troelstrup B. Functional and morphologic asymmetry in children with unilateral cross-bite. J Dent Res 1975; 5(Suppl): 178S.

18. Pirttiniemi P, Kantomaa T, Lahtela P. Relationship between craniofacial and condyle path asymmetry in unilateral crossbite patients. Eur J Orthod 1990; 12: 408-413.

19. ENLOw DH. Handbook of facial growth. Philadelphia: WB Saunders Company, 1986. 419-430.

20. LAm P, SAdowsky C, Omerza F. Mandibular asymmetry and condylar position in children with unilateral posterior crossbite. Am J Orthod Dentofacial Orthop 1999; 115: 569-575.

21. KiKi A, Kilic N, OKtay H. Condylar asymmetry in bilateral posterior crossbite patients. Angle Orthod 2007; 77: 77-81.

22. Alarcon JA, Martin C, Palma JC. Effect of unilateral posterior crossbite on the electromyographic activity of human masticatory muscles. Am J Orthod Dentofacial Orthop 2000; 118: $328-334$

23. Troelstrup B, Moller E. Electromyography of the temporalis and masseter muscles in children with unilateral crossbite. Scand J Dent Res 1970; 78: 425-430.

24. Ferrario VF, Sforza C, Serrao G. The influence of crossbite on the coordinated electromyographic activity of human 
masticatory muscles during mastication. J Oral Rehabil 1999; 26: $575-581$.

25. Sonnesen L, Bakke M, Solow B. Bite force in pre-orthodontic children with unilateral posterior crossbite. Eur J Orthod 2001; 23: 741-749.

26. Kiliaridis S, Mahboubi PH, RaAdsheer MC, Katsaros C. Ultrasonographic thickness of the masseter muscle in growing individuals with unilateral crossbite. Angle Orthod 2007; 77: 607-611.

27. JANKELSON B. Measurement accuracy of the mandibular kinesiograph - a computerized study. J Prosthet Dent 1980; 44: 656-666.

28. Castroflorio T, Farina D, Bottin A, Piancino MG, Bracco P, Merletti R. Surface EMG of jaw elevator muscles: effect of electrode location and inter-electrode distance. J Oral Rehabil 2005; 32: 708-713.

29. Lund JP, Scott G, Kolta A, Westberg GR. Role of cortical inputs and brainstem interneuron populations in patterning mastication. In: Nakamura Y, Sessle GJ, eds. Neurobiology of mastication. From molecular to system approach. Tokyo: Elsevier Science, 1999; 504-514.

30. IshiI N, Soma K, Toda K. Response properties of periodontal mechanorecptors in rats, in vitro. Brain Res Bull 2002; 58: 357 361.

31. Tsuboi A, Kolta A, Chen CC, Lund JP. Neurons of the trigeminal main sensory nucleus participate in the generation of rhythmic motor patterns. Eur J Neurosci 2003; 17: 229-238.

32. JohnSen S, TRULSSON M. Receptive field properties of human periodontal afferents responding to loading of premolar and molar teeth. J Neurophysiol 2003; 89: 1478-1487.
33. Lund JP, Kolta A. Generation of the central masticatory pattern and its modification by sensory feedback. Dysphagia 2006; 21: 167-174.

34. Yokomizo Y, Murai Y, Tanaka E, InOKichi H, Kusukawa J, Higashi H. Excitatory GABAergic synaptic potentials in the mesencephalic trigeminal nucleus of adult rat in vitro. Neurosci Res 2005; 51: 463-474.

35. Pinto AS, Buschang PH, Throckmorton GS, Chen P. Morphological and positional asymmetries of young children with functional unilateral posterior crossbite. Am J Orthod Dentofacial Orthop 2001; 120: 513-520.

36. Yoshino K, Kawagishi S, Takatsuki Y, Amano N. Different roles of the primary motor and ventral premotor cortex in jaw movements. In: Nakamura Y, Sessle GJ, eds. Neurobiology of masticatio. From molecular to system approach. Tokyo: Elsevier Science, 1999; 515-517.

37. Mioche L, Bourdiol P, Monier S. Chewing behaviour and bolus formation during mastication of meat with different textures. Arch Oral Biol 2003; 48: 193-200.

38. Piancino MG, Farina D, Talpone F, Castroflorio T, Gassino G, Margarino V, Bracco P. Surface emg of jawelevator muscles and chewing pattern in complete denture wearers. J Oral Rehabil 2005; 32: 863-870.

39. Petren S, Bondemark L, Soderfeldt B. A systemetic review concerning early orthodontic treatment of unilateral posterior crossbite. Angle Orthod 2003; 73: 588-596.

40. Kecik D, Kocadereli I, SaAtci I. Evaluation of the treatment changes of functional posterior crossbite in the mixed dentition. Am J Orthod Dentofacial Orthop 2007; 131: 202215. 